\title{
Analyse De Vulnérabilités Des Systèmes Agraires De La Région De La Boucle Du Mouhoun Au Burkina Faso
}

\author{
Joachim Bonkoungou \\ Chargé de recherches en géographie, INERA/CREAF Kamboinsé, \\ Burkina Faso \\ Jérôme Compaore
}

Chargé de recherches en communication, INERA/CREAF Kamboinsé, Burkina Faso

Farid Traore

Chargé de recherches en science et gestion de l'environnement, INERA/CREAF Kamboinsé, Burkina Faso

\section{Olivier Beucher}

Consultant, Baastel

\section{Issa Bikienga}

Consultant, ICI Burkina Faso

\section{Résumé}

Description du sujet: L'article a trait à des évaluations de la vulnérabilité au climat, pris dans le sens du rapport 4 du GIEC, incluant la sensibilité (aléas climatiques et exposition) et la capacité d'adaptation. La région d'étude est les systèmes agraires de la Boucle du Mouhoun (RBM).

Objectifs : L'objectif général poursuivi est d'analyser les vulnérabilités des systèmes agraires de la RBM. Les objectifs spécifiques ont été de faire un zonage des systèmes agraires de la RBM et d'évaluer, avec la participation active des parties prenantes, les vulnérabilités des systèmes agraires définis.

Méthodes : La revue documentaire, les ateliers d'appropriation des résultats, de collecte et de validation de données et les entretiens de groupes-cibles dans retenus, à partir d'une fiche d'évaluation de vulnérabilité, ont été utilisés.

Résultats : Les entretiens ont eu lieu dans 17 localités de la RBM. Quatre systèmes agraires, Sud-Sahélien, Vallée du Sourou, Nord -Soudanien et SudSoudanien, de niveaux différents de vulnérabilité, caractérisent la région d'étude. 
Conclusions : La disponibilité de l'eau en est le facteur déterminant. Alors, les zones situées plus au Nord, à pluviométries plus faibles, sont les plus vulnérables. De même, la Vallée du Sourou est le moins vulnérable du fait de la vallée du Sourou, d'un barrage-vanne et d'un canal de dérivation qui donnent de l'eau pour les activités de production agricoles durant toute l'année. En moyenne tous les systèmes agraires ont placé les aléas climatiques actuels comme le premier facteur qui va impacter leurs moyens de subsistance Les régions plus pluvieuses craignent les aléas climatiques à venir pendant que les régions moins pluvieuses redoutent plus les aléas climatiques actuels.

Mots clés: Vulnérabilités, changements climatiques, systèmes agraires, Région de la Boucle du Mouhoun, Burkina Faso

\title{
Vulnerability Analysis of the Boucle Du Mouhoun Region's Agrarian Systems in Burkina Faso
}

\author{
Joachim Bonkoungou \\ Chargé de recherches en géographie, INERA/CREAF Kamboinsé, \\ Burkina Faso \\ Jérôme Compaore \\ Chargé de recherches en communication, INERA/CREAF Kamboinsé, \\ Burkina Faso \\ Farid Traore \\ Chargé de recherches en science et gestion de l'environnement, \\ INERA/CREAF Kamboinsé, Burkina Faso \\ Olivier Beucher \\ Consultant, Baastel \\ Issa Bikienga \\ Consultant, ICI Burkina Faso
}

\begin{abstract}
Description: The article addresses climate vulnerability assessments, along the lines of IPCC Report 4, including sensitivity (climate hazards and exposure) and adaptive capacity. The study area is the agrarian systems of Boucle du Mouhoun Region (BMR).
\end{abstract}


Objectives: The general objective pursued is to analyze the vulnerabilities of agrarian systems of the RBM. The specific objectives were to do a zoning of BMR agrarian systems and to evaluate, with the active participation of stakeholders, the vulnerabilities of the defined agrarian systems.

Methods: Literature review, results appropriation, data collection and validation workshops, and target group interviews were selected from a vulnerability assessment sheet.

Results: Interviews took place in 17 localities of the BMR. Four agrarian systems, South Sahelian, Sourou Valley, North Sudan and South Sudan, with different levels of vulnerability, characterize the study area.

Conclusions: Availability of water is the determining factor. So the more northerly areas, with lower rainfall, are the most vulnerable. Similarly, the Sourou Valley is the least vulnerable because of the Sourou Valley, a Vann dam and a diversion channel that provide water for agricultural production activities throughout the year. On average, all agrarian systems have placed the current climatic hazards as the first factor that will impact their livelihoods The rainier regions are afraid of future climatic hazards while the less rainy regions are more afraid of the current climatic hazards..

Keywords: Vulnerabilities, climate change, agrarian systems, Boucle du Mouhoun Region, Burkina Faso

\section{Introduction}

L'Afrique, particulièrement les pays sahéliens à saisons alternées, sont très vulnérables aux changements climatiques (African Ministry Confenrence on Environment (AMCEN) 2014; Diallo 2012; Sultan et al. 2012). Pourtant c'est l'un des continents à faibles émissions de gaz-à-effet de serre, la cause majeure du réchauffement global (Groupe Intergouvernemental sur l'Evolution du Climat (GIEC) 2014a). Elle doit cette vulnérabilité à ses faibles capacités d'adaptation. Les nations africaines sont, dans la majeure partie, classées parmi les pays les moins avancées. Les populations africaines sont pauvres et n'ont pas les moyens pour s'adapter convenablement aux effets adverses des changements climatiques (Holmberg 2008; Mosha 2011; Turner 2010). La planification de l'adaptation devient alors une priorité pour se doter de capacités nécessaires.

Les gouvernements ont alors pris des engagements pour formuler et mettre en œuvre des programmes d'action nationaux d'adaptation (PANA). Le Burkina Faso, pays sahélien d'Afrique de l'Ouest, a adopté son PANA en 2007 qu'il a mis en œuvre, à travers des projets dans des sites pilotes, jusqu'en 2011-2012 (MECV 2007). Les résultats probants obtenus de ces PANA a nécessité la formulation d'un Plan National d'Adaptation (PNA) qui prend en compte tous les secteurs économiques exposés au climat. Un PNA 
d'ensemble a été synthétisé pour prendre en compte tous les secteurs économiques vulnérables en 2015 (Ministère de l'Environnement et des Ressources Halieutiques (MERH) 2015). L'un des reproches adressés à ce PNA est son caractère global. Les spécificités régionales n'ont pas été prises en compte. Dans l'optique d'y apporter une réponse, il a été initié au Burkina Faso, par l'Agence Française de Développement AFD), une étude de la vulnérabilité des zones rurales de la région de la Boucle du Mouhoun (RBM) aux changements climatiques. La présente étude devrait donner une réponse à la question de recherche suivante: quelles sont les vulnérabilités climatiques des systèmes agraires de la RBM au Burkina Faso ?

Il faut signaler que la RBM est une région d'intervention de l'AFD qui est un partenaire technique et financier de la Société des Fibres et Textiles (SOFITEX). L'étude s'était fixée pour objectif global d'analyser les vulnérabilités climatiques des systèmes agraires de la RBM. Les systèmes agraires de la région ont des degrés différents de vulnérabilités. La partie cidessous a trait à la méthodologie utilisée pour vérifier cette hypothèse.

\section{Méthodologie}

L'étude a été conduite par une équipe pluridisciplinaire d'experts qui ont utilisé plusieurs outils méthodologiques.

\subsection{Outils méthodologiques utilisés}

Plusieurs outils méthodologiques ont été utilisés pour la collecte des données et leur analyse. Un brainstorming a été utilisé par l'équipe afin de lister tous les services techniques, les organisations non-gouvernementales (ONG), les projets, les programmes, les organisations de la société civile, les organisations paysannes et les structures privés à visiter. Cette liste a été présentée et complétée lors de rencontres d'appropriation par les membres d'un comité multisectoriel de suivi de l'étude mis en place.

L'équipe s'est scindée en deux groupes afin de couvrir le maximum de services listés. Un guide d'entretien a servi pour la collecte des documents numériques et/ou analogiques disponibles, notamment les rapports et les publications scientifiques, ainsi que des données socioéconomiques pertinentes qui seront utiles pour l'analyse. Un répertoire ouvert sur Dropbox était accessible à tous les membres de l'équipe qui pouvaient y poster et consulter les documents numériques, les données et les rapports d'entretiens réalisés.

Le choix des villages pour les entretiens de groupes-cibles a été fait lors d'une réunion dans le chef-lieu de la région à Dédougou où des représentants des parties prenantes ont été conviés. Les systèmes agraires dans la région ont été délimités grâce à une cartographie participative. Une carte de la RBM 
imprimée a servi de support pour les ajouts apportés par les participants à l'atelier. Les entretiens dans les villages, en focus groupe de discussion, ont été conduits grâce à un outil d'évaluation de la réduction de la vulnérabilité. L'évaluation devrait se faire sur les quatre éléments suivants :

- Vulnérabilité des moyens de subsistance et du bien-être social au climat et à la variabilité climatique actuels ;

- Vulnérabilité des moyens de subsistance et du bien-être social aux risques climatiques à venir ;

- Obstacles et opportunités d'adaptation ;

- Capacité et volonté des communautés et acteurs des zones rurales à mettre en œuvre des mesures d'adaptation sur le long terme.

Une grille d'évaluation a été adoptée pour conduire ces évaluations participatives de vulnérabilités : 1 (pas vulnérable), 2 (peu vulnérable), 3 (moyennement vulnérable), 4 (Vulnérable) et 5 (Très vulnérable).

\subsection{Sites d'évaluation de la vulnérabilité}

La carte ci-dessous localise les villages visités pour l'évaluation de la vulnérabilité :

Figure 1: Sites d'étude

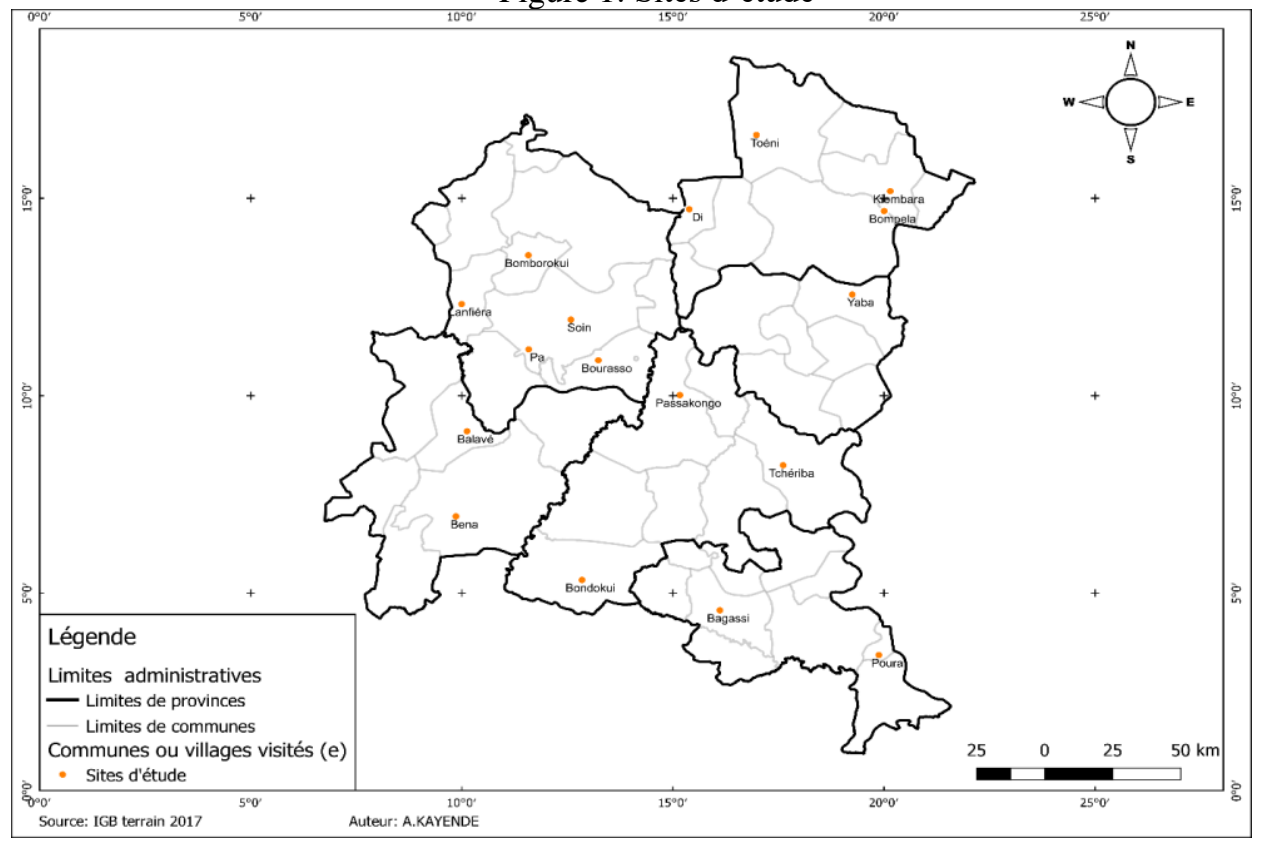

Les critères de choix de ces villages ont été la représentativité des systèmes agraires dans lesquels ils sont situés et leur accessibilité. Ainsi, les grands axes routiers ont été privilégiés dans le choix, de même que les chefslieux de département. 


\section{Résultats}

L'évaluation des vulnérabilités climatiques a été faite dans 17 localités de la RBM, à raison de 6 villages pour le système agraire SudSahélien, 2 pour la vallée du Sourou, 4 pour le Nord-Soudanien et 5 pour le Sud-Soudanien. Les résultats sont organisés autour des systèmes agraires et de leurs vulnérabilités aux changements climatiques.

\subsection{Systèmes agraires de la RBM}

Deux domaines climatiques se partagent la RBM. Il s'agit du domaine soudano-sahélien caractérisé par une pluviométrie moyenne annuelle qui fluctue entre 600 et $900 \mathrm{~mm}$ d'eau. La saison des pluies s'étale en moyenne sur une durée de 3 mois à 6 mois. Ce domaine climatique couvre la majeure partie de la RBM, depuis le Sud jusqu'au Nord, ne laissant qu'une petite portion de territoire où sévit un climat plus rude, le domaine sahélien. Les pluviométries moyennes annuelles y sont plus faibles, entre 300 de $600 \mathrm{~mm}$ en moyenne par an. La saison des pluies a également une durée moyenne plus courte de moins de 3 mois. Ce sont des climats tropicaux caractérisés par la forte variabilité spatiotemporelle des précipitations. Cette pluviométrie détermine les systèmes agraires.

Quatre zones agraires ont été définies (Carte 2): la zone SudSahélienne et la zone aménagée de la vallée du Sourou dans la partie septentrionale et les zones Nord-Soudanienne et Sud-Soudanienne dans la partie méridionale de la RBM.

La zone agraire Sud-Sahélienne est dominée par le système de céréales sèches, notamment le mil et le sorgho. Les céréales occupent $75 \%$ des superficies cultivées dans la zone. Il s'y produit également le sésame, la principale culture de rente. Les fins brusques actuelles des saisons des pluies amènent des paysans à l'abandon de cette culture. On y pratique un élevage sédentaire dominé par les caprins et le pastoralisme. Cette zone dispose de deux zones pastorales à Barani et Toéni. Le taux d'équipement est de l'ordre de $40 \%$ avec un accès faible aux intrants. Le système agraire Sud-Sahélien étant dominé par les cultures céréalières, l'accès au crédit est par conséquent très limité. Du fait du caractère erratique des précipitations, les producteurs mettent en œuvre des mesures de conservation des eaux et des sols. Il s'agit principalement du Zaï et des cordons pierreux. Les producteurs utilisent principalement de la fumure organique et se contentent des engrais subventionnés par l'Etat, en très faibles quantités.

La zone agraire aménagée de la Vallée du Sourou est largement avantagée par la rivière dudit nom, de $120 \mathrm{~km}$, un affluent du Mouhoun, le plus important fleuve du pays. Un barrage-vanne et un canal de dérivation ont été construits à Léry pour rendre l'eau plus disponible pour les productions agricoles, en toutes saisons. C'est une zone d'irrigation où 288 
ha ont été aménagés de 1976 à 1983 à Guièdougou, puis 1000 autres ha sous la période révolutionnaire 1983-1990, 3000 ha sous l'ère libérale amorcée en 1991 avec le vote de la constitution et 2240 ha par le Millenium Challenge Account des Etats-Unis d'Amérique, entre 2008 et 2014. Les anciens périmètres rencontrent actuellement d'énormes difficultés du fait de la vétusté des installations. Des producteurs privés y ont faits leurs propres aménagements hors périmètres et disposent de motopompes pour amener l'eau à leurs parcelles. Le système de culture se compose de cultures maraichères et du maïs. Trois campagnes de production sont observées grâce à l'irrigation. L'oignon, qui se conserve plus facilement, est plus produit au détriment de la tomate. Durant les pics de production, les prix aux producteurs de la tomate peuvent baisser du simple au triple. Les producteurs n'arrivent même pas, dans ce cas, à couvrir les dépenses qui ont été engagées.

Figure 2: Les systèmes agraires de la RBM

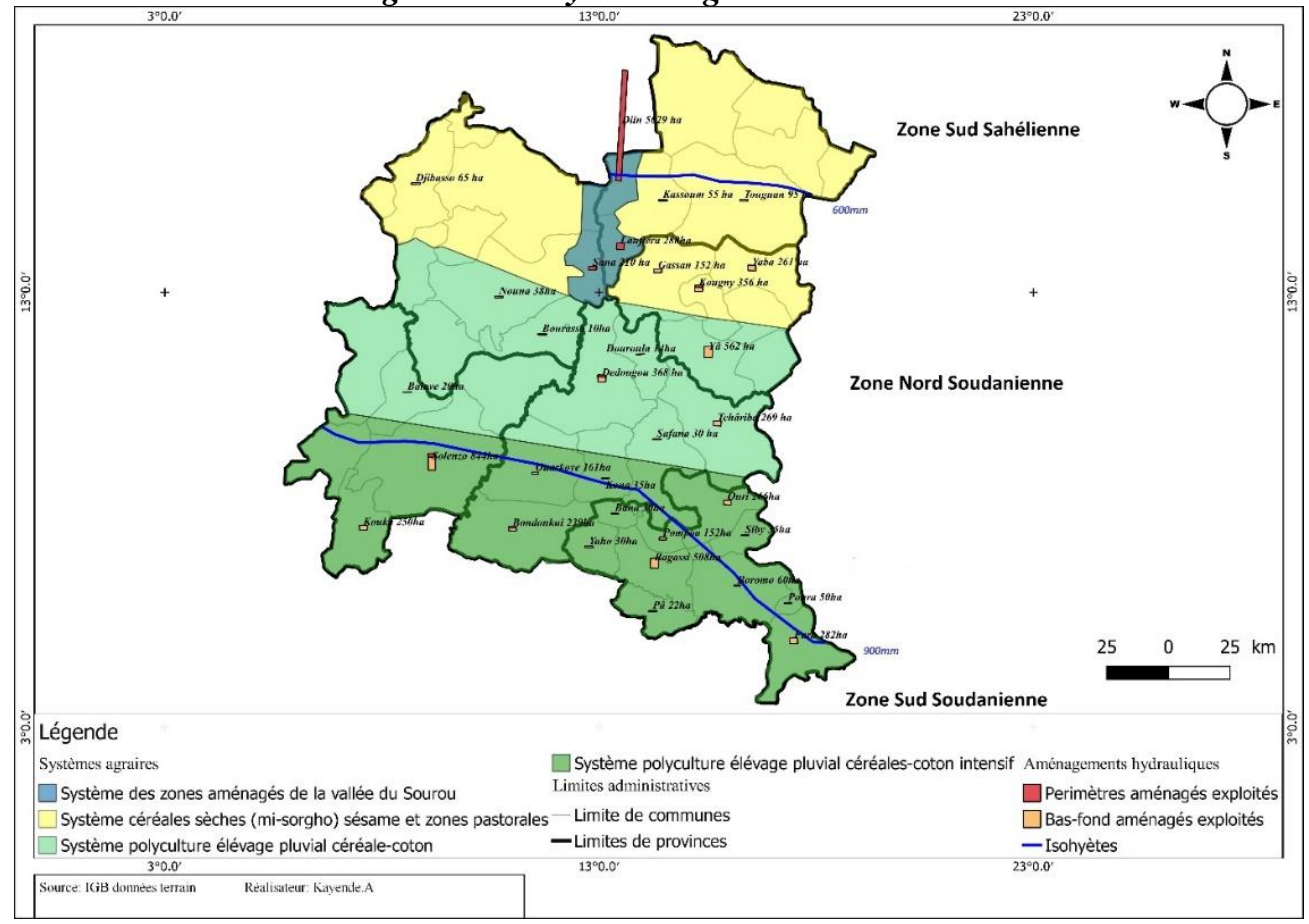

Les zones Nord-Soudanienne et Sud-Soudanienne sont les plus arrosées de la RBM. Il y tombe en moyenne 700 à $900 \mathrm{~mm}$ d'eau par an. C'est une région qui a bénéficié d'importantes vagues d'immigration occasionnées par les sécheresses des années 1970, de populations venues principalement des régions plus arides du Nord. Certains villages comptent près de $80 \%$ d'immigrants selon les estimations faites. Ces immigrants ont importé dans cette zone de nouveaux systèmes de production. Les systèmes de cultures 
actuels sont faits de rotation de cultures vivrières, de maïs et de sorgho, et de culture du coton, introduit par l'Etat pour des besoins d'entrée de devises étrangères. La production de coton est encadrée et bénéficie d'avantages que les autres cultures n’ont pas. Les intrants, composés de semences améliorées, d'herbicides et d'engrais, sont distribués sous forme de prêts remboursables à la vente, par la Société des Fibres et Textiles, 1'une des trois sociétés cotonnières implantées dans le pays. C'est pourquoi, le taux d'équipement est plus important et avoisine $80 \%$. C'est une zone d'élevage sédentaire et de transhumance, à l'intérieur de la région et vers le Sud, dans les régions administratives du Sud-Ouest et des Hauts-Bassins, la Côte d'Ivoire et le Ghana. Des transhumants, venus des contrées plus sèches, se sont établis dans la RBM. La monétarisation de l'économie a engendré la multiplication de petites exploitations et la création de coopératives pour rendre l'accès aux crédits plus faciles. Des structures privées et/ou traditionnelles octroient aussi des crédits aux producteurs.

\subsection{Vulnérabilités aux changements climatiques}

Les notations ont été faites dans chacun des villages listés sur la carte des sites. Les moyennes ont été calculées pour chaque systèmes agraires. Les résultats de vulnérabilités aux changements climatiques sont présentés selon la région et les différents systèmes agraires.

\subsubsection{Vulnérabilités de la RBM}

La figure ci-dessous retrace les niveaux moyens de vulnérabilité évalués conjointement avec les populations.

Figure 3 : Vulnérabilités de la RBM aux changements climatiques

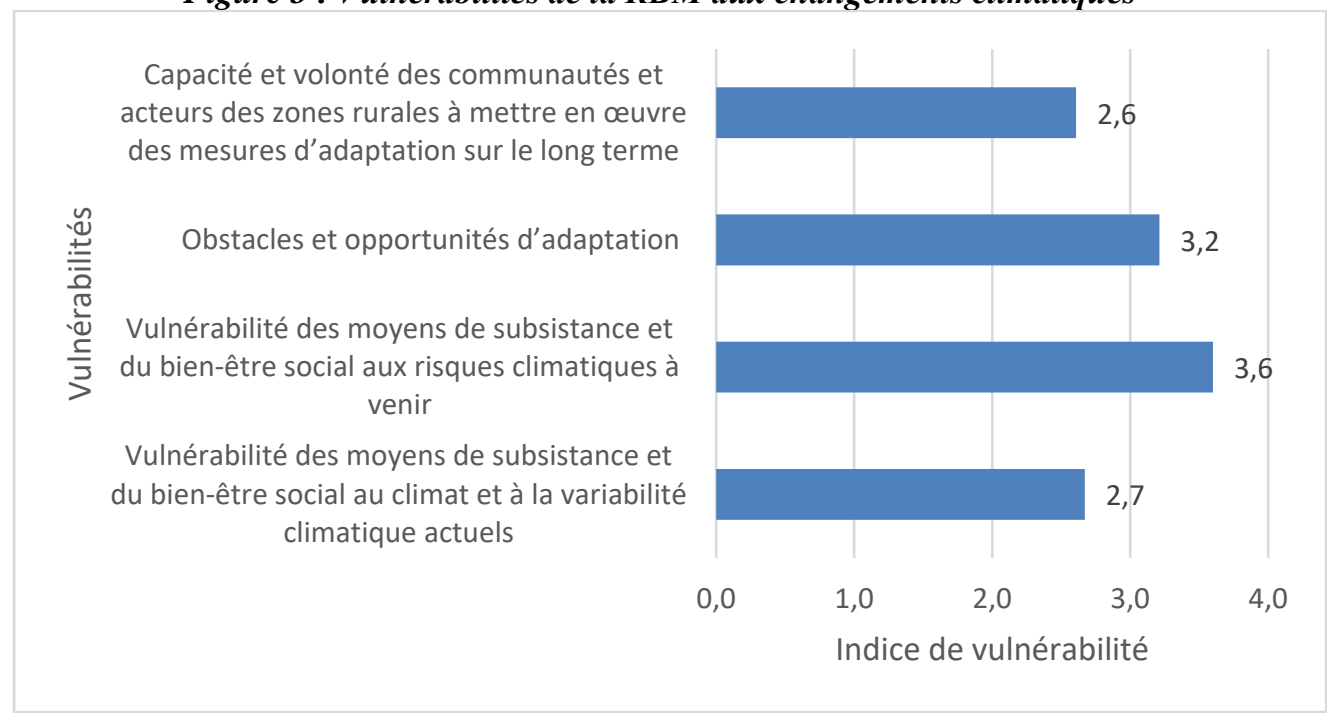


La lecture de la figure 3 montre bien que les producteurs de la RBM redoutent beaucoup plus les risques climatiques à venir, les inondations et les sécheresses notamment, qui vont rendre vulnérables leurs moyens de subsistance et de bien-être. Cette vulnérabilité a obtenu la moyenne des notes la plus élevée de 3,6. Elle est suivie par les obstacles et les opportunités d'adaptation dont disposent les producteurs qui a obtenu une moyenne de 3,2 . Les deux dernières vulnérabilités ont obtenu des moyennes sensiblement égales : 2,7 pour la vulnérabilité des moyens d'existence et du bien-être social au climat et à la variabilité climatique actuels et 2,6 pour les capacités et volonté des communautés et acteurs à mettre en œuvre des mesures d'adaptation sur le long terme.

\subsubsection{Vulnérabilités des systèmes agraires}

La figure 4 montre que le système du Sourou est de loin le moins vulnérable. Son indice de vulnérabilité moyen est 2,25 points, suivi du système Sud-Soudanien 3,18 et du Système Nord-Soudanien 3,25. Le plus vulnérable est le système Sud-Sahélien avec une moyenne de 3,42. En dehors du Sourou où l'eau ne constitue pas un facteur majeur de production, la vulnérabilité suit ici un gradient nord-sud. Plus on va vers le nord, plus la vulnérabilité devient importante. Les précipitations suivent ce même gradient. Elles sont plus abondantes au Sud et moins abondantes au Nord.

La vulnérabilité d'ensemble, prenant en compte les quatre vulnérabilités ci-dessus, est de 3,0. Pour les populations, la RBM est vulnérable aux changements climatiques.

Figure 4 : Vulnérabilités des systèmes agraires de la RBM

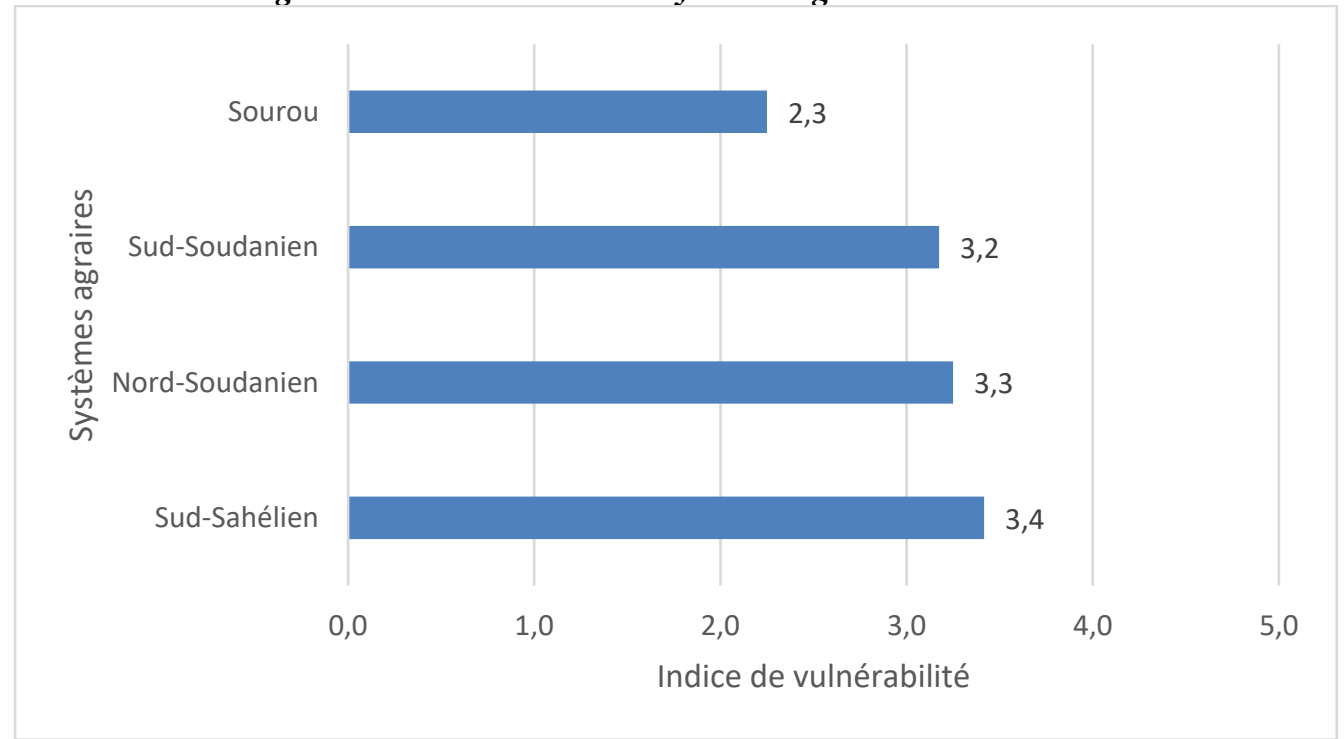




\subsubsection{Détail de la vulnérabilité des systèmes agraires}

Dans les détails, (figure 5), on peut remarquer que la vulnérabilité pour l'avenir occupe la première place pour les systèmes agraires SudSahélien et Nord-Soudanien. Dans le système agraire sud-Soudanien et du Sourou, c'est la vulnérabilité liée aux obstacles et opportunités d'adaptation que les populations des zones rurales craignaient le plus. Elle y est secondée par les vulnérabilités futures et constitue la seconde vulnérabilité dans le système Sud-Soudanien et la troisième dans le Sud-Sahélien où la deuxième vulnérabilité vulnérabilités au climat et à la variabilité actuelle du climat. Dans les autres systèmes agraires, cette dernière vulnérabilité est la moins redoutée des populations rurales. Dans le système agraire du Sourou, elle a obtenu une moyenne inférieure à 2 points d'indice de vulnérabilité.

Figure 5 : Détail des vulnérabilités des systèmes agraires

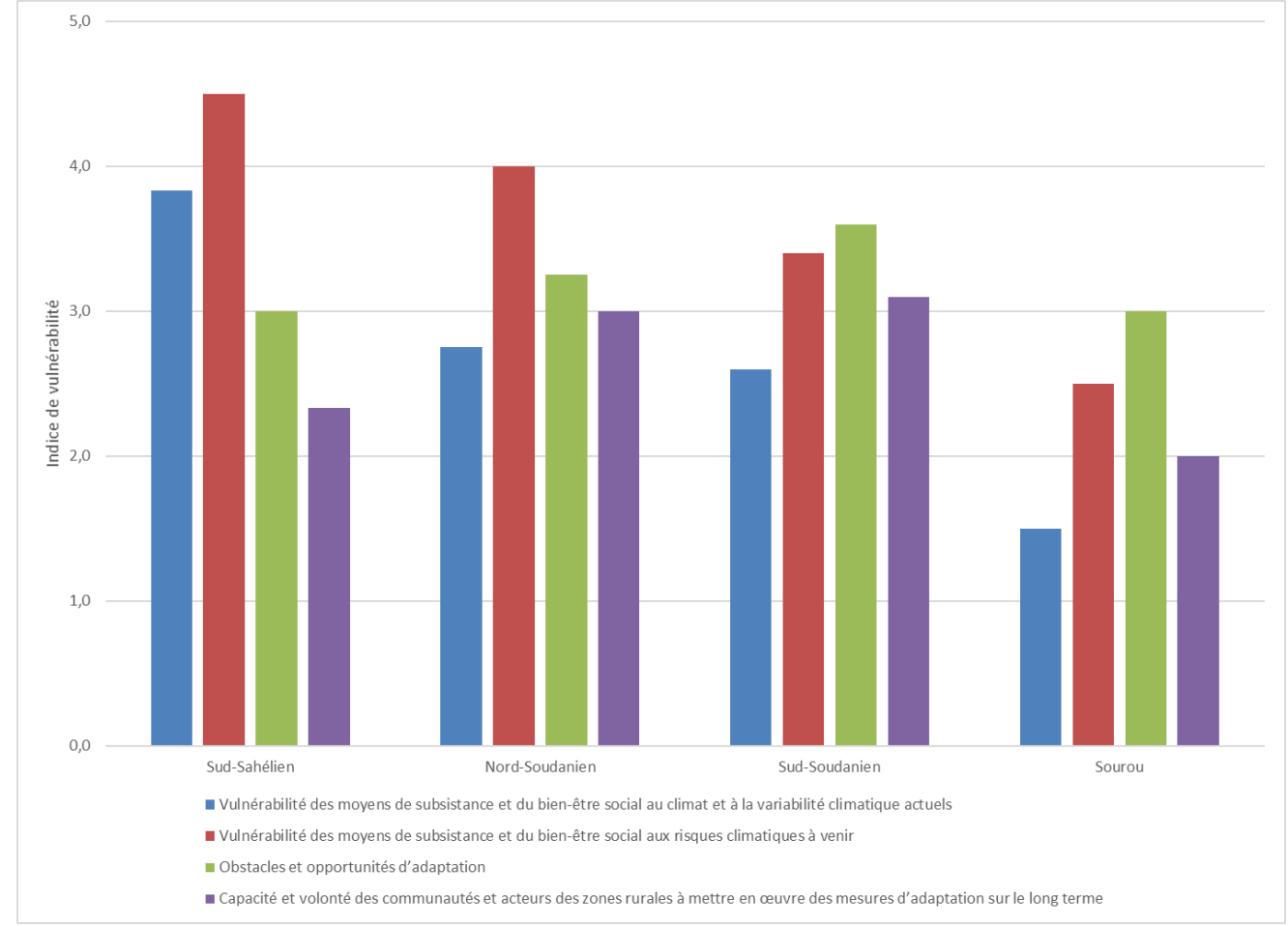

\section{Discussions}

La vulnérabilité est déterminée par les moyens de subsistance mis en œuvre par les producteurs mais aussi par d'autres facteurs importants dont le niveau de pauvreté. Les moyens de subsistance en question sont l'agriculture et l'élevage. L'eau est le facteur limitant déterminant la vulnérabilité au climat 


\subsection{L'agriculture}

Les moyens de subsistance des milieux ruraux de la RBM sont principalement l'agriculture et l'élevage. Chacun de ces moyens de subsistance peut être subdivisé en deux sous-groupes. L'activité largement dominante reste de loin l'agriculture pluviale, qui se déroule durant la saison des pluies, dont les durées vont de plus de 6 mois au sud à moins de 3 mois au nord de la RBM. C'est une activité dont les performances sont étroitement liées aux aléas et changements climatiques (Aggarwal and al. 2013; Mubaya 2010; Scheraga 2010). Il s'agit principalement des poches de sécheresse, des inondations provoquées par les fortes et successives pluies, de l'installation capricieuse des saisons et aussi de leurs brusques fins. Ces aléas et changements climatiques causent de mauvais rendements agricoles (Banque Mondiale (BM) 2011; Brown and Hansen 2008; Cooper et al. 2013; Tennigkeit et al. 2013).

Les années de mauvaises pluviométries sont en effet marquées par de faibles productivités. Les productions agricoles n'arrivent pas à couvrir les besoins alimentaires des populations et il est fait recours à des importations de vivres. Ce fut le cas de la saison agricole 2017-2018 où les pluies irrégulières ont fait chuter la production agricole, obligeant les autorités nationales à faire appel au stock de sécurité de la Communauté Economiques des Etats de l'Afrique de l'Ouest. Des filets sociaux sont mis en œuvre sur le terrain pour éviter que les producteurs les plus démunis perdent leurs moyens de subsistance (Burkina Faso 2015; FAD 2010; Gubbels 2011; Hourticq and al. 2013; Ministère de l'Environnement et des Ressources Halieutiques (MERH) 2015).

La production de coton a donné également de mauvais résultats. Les baisses de production sont dues entre autres à l'abandon du coton transgénique $\mathrm{Bt}^{1}$ au profit de coton conventionnel au potentiel de production plus faible. Elles ont été provoquées par une mauvaise saison des pluies marquées par une irrégularité des pluies. Les revenus des producteurs de coton, regroupés dans des coopératives et groupements, n'ont pas alors suffi à rembourser les crédits alloués sous forme d'intrants agricoles par la SOFITEX. Ces derniers se retrouvent alors endettés (Poisot and Zoundi 2007; SNV 2014). A leurs yeux, la production de coton est source d'endettement et de paupérisation. Elle a conduit à des conflits entre la société cotonnière et une grande partie des producteurs qui refusent maintenant de s'adonner à la culture du coton. Cette dernière a même déposé une plainte en justice qui suit son cours.

Durant la longue saison sèche, notamment dans la Vallée du Sourou, il se pratique l'irrigation. La maitrise partielle ou totale de l'eau à des fins de

${ }^{1}$ Bacillus thuringiensis 
productions agricoles occasionne des rendements plus importants que dans le système pluvial et génère plus de revenus pour les producteurs (Barbier et al. 2011; Enee 2007; Griffon et al. 2001; WARDA 2005). Ces derniers font face cependant à une faible diversification des productions. Pendant les pics de production, notamment de la tomate et des productions agricoles de conservation difficile, les prix de vente sont moins intéressants. On peut assister à des destructions des productions et à des ventes à perte (Broutin and al. 2014; IED 2014).

\subsection{L'élevage}

Il existe aussi deux formes d'élevage qui sont pratiquées dans la RBM : l'élevage sédentaire et l'élevage transhumant. A l'instar de l'agriculture pluviale, les deux types d'élevage sont extensifs (Faye 2011). L'élevage sédentaire est pratiqué par la quasi-totalité des ménages. Le bétail est une forme de thésaurisation pour les habitants (Bonkoungou 2007; Diouf 2008; Grûnewaki 2012; Mahamadou 2011). Il est vendu à tout moment, principalement pendant les périodes difficiles, pour faire face aux dépenses urgentes comme la scolarité des enfants, les soins de santé et même pour l'achat de vivres. Les jours de marché sont les périodes propices pour l'écoulement. Le gros bétail, notamment les ânes et les bœufs sont élevés pour les besoins d'attelage avec des charrues, des charrettes et des brouettes. C'est une intégration de l'agriculture et de l'élevage (FAO 2013; Ouédraogo 2012; Scholle 2015).

L'élevage transhumant est plutôt pratiqué par des peuples nomades dont une partie s'est installée dans la RBM. C'est une forme traditionnelle d'adaptation aux changements climatiques (Agripade 2016; Bonkoungou 2015; Cissé et al. 2010; Touré et al. 2011). Les troupeaux sont déplacés au gré des saisons, à la recherche d'eau et de pâturage. Les circuits de transhumance sont internes dans la RBM et/ou externes en direction du sud. Des zones de pâturage drainent également un nombre important d'animaux. De plus en plus, les éleveurs transhumants convoitent les zones protégées qui disposent plus de ressources naturelles. Les pistes de bétail longent principalement les cours d'eau. Les animaux sont confrontés par conséquent à de nombreuses maladies et causent souvent des dégâts et des conflits avec les agriculteurs sédentaires (Deynès 2008; Djiga 2009; Fluet 2006; Hellendorff 2012; Kaboré 2009; PNUE 2011).

\subsection{L'eau, principal facteur limitant}

Le degré de vulnérabilité semble être en relation étroite avec la disponibilité de l'eau. Plus l'eau est disponible, plus la vulnérabilité est faible. Le gradient nord sud de la pluviométrie détermine par conséquent le niveau de vulnérabilité. Les régions du sud, plus arrosées, ont le niveau le 
plus faible comparativement à celles du nord plus arides. Seule la vallée du Sourou vient rompre ce gradient de vulnérabilité. La disponibilité de l'eau toute l'année y autorise d'avoir au moins deux campagnes de récolte dans l'année.

C'est ce qui explique que les régions dont l'économie repose sur les secteurs primaires de production que sont l'agriculture et l'élevage soient les plus vulnérables aux changements climatiques. Ainsi, la RBM, à l'instar du pays et de l'Afrique, est une région vulnérable aux changements climatiques. Pourtant, pour le GIEC et de nombreux auteurs, l'Afrique doit plutôt sa vulnérabilité à sa faiblesse technique (Groupe Intergouvernemental sur l'Evolution du Climat (GIEC) 2014b). Les populations et les gouvernements n'ont pas suffisamment de moyens financiers pour acquérir les moyens de production qui peuvent les rendre moins dépendants de l'offre pluviométrique.

La maitrise partielle et totale de l'eau participe à réduire la vulnérabilité. La RBM est pauvre en barrages et retenues d'eau qui sont plus implantés dans sa région nord où les pluies sont rares et mal réparties dans le temps et dans l'espace. Même dans les zones dites à faibles vulnérabilités, tous les exploitants ne présentent pas la même exposition. Ceux qui disposent de moyens efficaces d'exhaure de l'eau sont moins vulnérables que ceux qui en sont démunis. Ils peuvent utiliser les moyens dont ils disposent pour augmenter les superficies exploitées, diversifier leurs productions et bénéficier nettement mieux des marchés.

\section{Conclusion}

La RBM, à l'instar du Burkina Faso, est une région vulnérable aux changements climatiques. Les activités mises en œuvre, l'agriculture et l'élevage, ont des performances largement dépendantes des conditions pluviométriques. Les changements climatiques vont certainement exacerber cette vulnérabilité et compromettre l'évolution économique.

Cette vulnérabilité a été attribuée, pour les différentes sous-régions qui la composent, à la disponibilité de l'eau pour la mise en œuvre des activités du secteur primaire. Les parties qui reçoivent le plus gros volume de précipitations annuelles sont les moins vulnérables. Il en est de même des zones où des aménagements, comme les barrages et les retenues d'eau, rendent l'eau disponible en toutes périodes de l'année pour les activités de productions agricoles.

Des vulnérabilités différentes sont à établir dans chacune de ces zones où les populations ne disposent pas des moyens pour les réduire. Ainsi, dans toutes les zones, les habitants qui ne disposent d'outils pour mieux exploiter les ressources naturelles dont ils disposent devront avoir les niveaux les plus élevés de vulnérabilités. 


\section{Remerciements}

Cette publication a été tirée des travaux de consultation attribuée au groupe BAASTEL (www.baastel.com) et à ICI Burkina Faso, sur financement de l'Agence Française de Développement (https://www.afd.fr/fr), à qui nous adressons nos remerciements pour l'autorisation de publication qui nous a été donnée. Nos remerciements sont adressés à toutes les parties prenantes depuis l'échelle du ministère en charge de l'agriculture, aux populations des villages choisis, en passant par les autorités et la société civile de la région de la Boucle du Mouhoun.

\section{References:}

1. African Ministry Confenrence on Environment (AMCEN). 2014. Strategie Africaine Sur Les Changements Climatiques.

2. Aggarwal, P., Robert Zougmoré, and J. Kinyangi. 2013. ClimateSmart-Villages: A Community Approach to Sustainable Agricultural Development. Copenhagen, Denmark: CCAFS. Retrieved (www.ccafs.cgiar.org).

3. Agripade. 2016. Recherches Sur La Résilience Au Sénégal et Au Burkina Faso : Les Premières Leçons Du PRESA.

4. Banque Mondiale (BM). 2011. Burkina Faso Disaster Risk Management : Country Note.

5. Barbier, Bruno, Hervé Ouédraogo, Hamma Yacouba, Boubacar Barry, and Jean-yves Jamin. 2011. “L'agriculture Irriguée Dans Le Sahel Ouest-Africain. Diversité Des Pratiques et Des Performances." Cah Agric 20:24-33.

6. Bonkoungou, Joachim. 2007. “Zonage Des Potentialités Agricoles Du Bassin Versant Pilote Du Zondoma, Burkina Faso." Centre Régional AGRHYMET, Niamey, Niger.

7. Bonkoungou, Joachim. 2015. "Variabilites, Changements Climatiques et Vulnerabilites Des Populations Au Burkina Faso." Thèse de doctorat de géographie, Univ. Abdou Moumouni, Niamey, Niger

8. Broutin, C., B. Hermelin, and L. Levard. 2014. Comment Améliorer l'accès Au Marché Pour Les Exploitations Familiales?

9. Brown, Casey and James W. Hansen. 2008. Agricultural Water Management and Climate Risk. New-York, USA. Retrieved (http://iri.columbia.edu/publications/search.php?id=780).

10. Burkina Faso. 2015. Plan National de Développement Économique et Social. Ouagadougou, Burkina Faso.

11. Cissé, Pierre, Zorom Malicki, Bruno Barbier, and Abdou Maïga. 2010. “Les Migrations, Une Stratégie d'adaptation à La Variabilité 
Climatique En Zone Sahélienne.” RGLL 8:184-96.

12. Cooper, Peter J. M. et al. 2013. Large-Scale Implementation of Adaptation and Mitigation Actions in Agriculture. Copenhagen, Denmark. Retrieved (www.ccafs.cgiar.org).

13. Deynès, Fabien. 2008. Les Impacts Du Changement Climatique En Afrique : Conflictualité Associée.

14. Diallo, Bintou. 2012. "Etude de La Vulnérabilité et de l'adaptation $\mathrm{Au}$ Changement Climatique: Cas Des Sites Pilotes Du Projet PRGDT Au Burkina Faso." CRA Niamey, Niger.

15. Diouf, Aliou. 2008. Interactions Société, Nature et Climat Au Sahel: La Rupture Socio-Économique et Écologique Au Centre-Est AgroSylvopastoral Sénégalais Du XXe Siècle.

16. Djiga, Habib Ahmed. 2009. "La Gouvernance Locale à l'épreuve Des Migrations Dues Aux Changements Climatiques: Cas Des Rapports Entre Conseils Villageois de Développement et Chefs Traditionnels Au Burkina Faso Dans La Gestion Des Conflits Sociaux." La Revue Électronique En Sciences de l'Environnement 6:6. Retrieved (http://id.erudit.org/iderudit/044565ar).

17. Enee, M. grégory. 2007. La Dynamique Des ONG Au Burkina Faso : Une Efficacité En Question.

18. FAD. 2010. Programme d'appui à La Stratégie de Réduction de La Pauvreté Au Burkina Faso : Rapport d'évaluation.

19. FAO. 2013. La Résilience Des Moyens d'existence. Réduction Des Risques de Catastrophe Pour La Sécurité Alimentaire et Nutritionnelle.

20. Faye, Mbaye Mbengue. 2011. Projet Pôle de Bagré: Plan Cadre de Gestion Environnementale et Sociale.

21. Fluet, Marie-Joël. 2006. "Impacts Des Changements Climatiques Sur Les Agriculteurs de La Province Du Zondoma Au Burkina Faso: Adaptation, Savoir et Vulnérabilité." Université du Québec à Montréal, Canada. Retrieved (http://www.archipel.uqam.ca/3513/).

22. Griffon, Michel et al. 2001. Filières Agroalimentaires En Afrique: Comment Rendre Le Marché plus Efficace.

23. Groupe Intergouvernemental sur l'Evolution du Climat (GIEC). 2014a. Changements Climatiques 2014 Incidences, Adaptation et Vulnérabilité. Résumé à l'intention Des Décideurs.

24. Groupe Intergouvernemental sur l'Evolution du Climat (GIEC). 2014b. "Emergent Risks and Key Vulnerabilities." Pp. 1039-99 in Climate cchange 2014.

25. Grûnewaki, François. 2012. Combattre l'insécurité Alimentaire Dans Les Contextes d'Etat Fragile et Les Situations de Crise et Post Crise.

26. Gubbels, Peter. 2011. Échapper Au Cycle de La Faim: Les Chemins 
de La Résilience Au Sahel.

27. Hellendorff, Par Bruno. 2012. "Changement Climatique et Conflits Agro-Pastoraux Au Sahel.” Note d'analyse Du GRIP 14. Retrieved (http://www.grip.org/fr/node/546).

28. Holmberg, Johan. 2008. "Natural Resources in Sub-Saharan Africa: Assets and Vulnerabilities." Current African Issues 39:52.

29. Hourticq, J., M. Taondyande, and S. Soubeiga. 2013. Revue Diagnostique Des Dépenses Publiques de Base Dans Le Secteur Agricole (2004-2012). Retrieved (http://wwwwds.worldbank.org/external/default/WDSContentServer/WDSP/IB/2 013/12/06/000333037_20131206160455/Rendered/PDF/831620WP0 FRENC00Box379886B00PUBLIC0.pdf).

30. IED. 2014. "Agriculture Familiale et Lutte Contre La Pauvreté." AGRIPADE 30(2):36.

31. Kaboré, Oumar. 2009. "Dynamique Des Plans d'eau Liée à La Vulnérabilité Climatique et à l'action de l'homme." Université de Ouagadougou, Burkina Faso.

32. Mahamadou, Illou. 2011. "Impacts Des Variations de La Crue Sur Les Communautés Rurales Du Delta Intérieur Du Bassin Versant Du Fleuve Niger." Université Abdou Moumouni, Niamey, Niger.

33. MECV. 2007. Plan d'action National d'adaptation Du Burkina Faso. Ouagadougou, Burkina Faso: MECV.

34. Ministère de l'Environnement et des Ressources Halieutiques (MERH). 2015. Plan National d'adaptation Aux Changements Climatiques Du Burkina Faso.

35. Mosha, a. C. 2011. "Climate Change and Poverty in Africa: An Overview." Regional Development Dialogue 32(1):1-30.

36. Mubaya, Chipo Plaxedes. 2010. "Farmer Strategies towards Climate Variability and Change in Zimbabwé and Zambia." University of the Free State Bloemfontein, South-Africa.

37. Ouédraogo, Lucien. 2012. “Gestion de 1'eau et Adaptation Des Populations Au Changement Climatique Dans Le Bassin Versant de Yakouta, Sahel Du Burkina Faso." Université Abdou Moumouni, Niamey, Niger.

38. PNUE. 2011. Sécurité Des Moyens d'existence. Changments Climatiques, Migrations et Conflits Au Sahel.

39. Poisot, Anne-Sophie and Jean Sibiri Zoundi. 2007. "Systèmes Agraires Durables, Vulnérabilité et Bonnes Pratiques Agricoles Dans l'Ouest Du Burkina Faso: Actes de l'atelier FAO-INERA Sur Les Bonnes Pratiques Agricoles." Pp. 18-20 in Actes atelier FAO-INERA sur les bonnes pratiques agricoles. Bobo-Dioulasso.

40. Scheraga, Joel D. 2010. An Introduction to Climate Change 


\section{Adaptation.}

41. Scholle, Justine. 2015. Pratiques Agroécologiques et Agroforestières En Zone Tropicale Humide.

42. SNV. 2014. Relever Ensemble Le Défi de La Sécurité Alimentaire Au Niger Revue Critique Des Relever Ensemble.

43. Sultan, Benjamin et al. 2012. "La Question de La Vulnérabilité et de l'adaptation de l'agriculture Sahélienne $\mathrm{Au}$ Climat $\mathrm{Au}$ Sein $\mathrm{Du}$ Programme AMMA.” Pp. 64-72 in La météorologie, Spécial AMMA.

44. Tennigkeit, Timm, Andreas Wilkes, Charlie Parker, and Fred Kossam. 2013. Climate Change and Agriculture in LDCs.

45. Touré, Hamidou et al. 2011. Etudes de Modélisation Climatique, d'évaluation Des Rsiques et d'analyse de La Vulnérabilité Aux Changements Climatiques: Données Sectorielles: Agriculture. Ouagadougou, Burkina Faso: Laboratoire d'Analyses Mathématiques des Equations, Université de Ouagadougou.

46. Turner, B. L. 2010. "Vulnerability and Resilience: Coalescing or Paralleling Approaches for Sustainability Science?" Global Environmental Change 20(4):570-76. Retrieved (http://dx.doi.org/10.1016/j.gloenvcha.2010.07.003).

47. WARDA. 2005. "Rice Policy and Food Security in Sub-Saharan Africa." P. 407 in, edited by P. Kormawa and A. A. Touré. Cotonou, Benin. 